\title{
Lumbar Pyogenic Spondylodiscitis and Bilateral Psoas Abscesses Extending to the Gluteal Muscles and Intrapelvic Area Treated with CT-guided Percutaneous Drainage - A Case Report -
}

\author{
Bong-Jin Lee*, Seong-Tae Kim, Kwon-Hee Park*, Jeong Heon Oh ${ }^{\dagger}, \mathrm{Hyun} \mathrm{Kim}^{\dagger}$ \\ Departments of * Orthopedic Surgery, ${ }^{\dagger}$ Internal Medicine, ${ }^{\dagger}$ Radiology, Cheju Halla General Hospital, Jeju, Korea
}

\begin{abstract}
Bilateral psoas abscesses extending to the gluteal muscle and intrapelvic area are uncommon. We present our experience with computed tomography (CT)-guided percutaneous catheter drainage for the treatment of multiple aggressive abscesses in a diabetic patient. The abscesses completely resolved after the procedures. Psoas abscess should be considered in the dif ferential diagnosis of older diabetic patients with fever, flank or back pain, and flexion contracture of the hip joint. CT scan ning is a useful method in diagnosing abscesses, and CT-guided percutaneous catheter drainage is an effective treatment method in selected patients.
\end{abstract}

Key Words: Psoas abscess extending into the gluteal muscle and intrapelvic area, Diabetic patient, CT-guided percutaneous drainage

\section{Introduction}

Psoas abscess is a potentially life-threatening diagnosis. It is classified as primary if there is no obvious source of infection, and as secondary if there is an apparent underlying cause. Prior to modern antituberculous therapy, tuberculous spondylitis was responsible for the majority of psoas abscesses. With the control of Mycobacterium tuberculosis infection, other disease entities have become leading causes of secondary psoas abscesses: gastrointestinal tract disease, including Crohn's disease; trauma; genito-urinary tract infection; and vertebral osteomyelitis ${ }^{1-4}$.

Primary psoas abscesses are likely to occur secondary to unrecognized septicemia in developing or tropical countries. Primary psoas abscesses are prevalent in intravenous drug abusers and in immunodeficient patients ${ }^{4}$. Concerning hematogenous dissemination, the psoas muscle may be at high risk for abscess formation because of the rich vascular supply from the spine and lymphatic channels overlying the muscle ${ }^{4}$. The case we present is considered a secondary psoas abscess. The patient also presented with early spondylodiscitis of L2-L3.

Bilateral psoas abscesses are rare and are diagnostically and therapeutically challenging because the presenting signs and symptoms are insidious and highly variable. To the best of our knowledge, bilateral and multiple psoas abscesses accompanying multilocular intrapelvic and gluteal abscesses are extremely rare. We describe our experience using CT-guided percutaneous catheter drainage for the treatment of multiple aggressive abscesses in a diabetic patient.

\section{Case Report}

A 54-year-old male patient was transferred from a local clinic, complaining of general weakness and lower back pain lasting for one week. The patient had a 10-year-history 
of diabetes mellitus and alcoholism with liver cirrhosis. Physical examination revealed multiple tender points on the back and both flanks and inguinal areas. Body temperature was $38^{\circ} \mathrm{C}$. The range of motion in his spine was severely limited, and flexion contractures were noted in both hip and knee joints.

T1-weighted coronal MRI showed multiple low-signal intensities in both psoas muscles (Fig. 1A). T2-weighted coronal MRI showed multiple high-signal intensities in both psoas muscles and multiple high-signal bone marrow infiltrations in the L2 and L3 bodies (Fig. 1B). Enhanced T1weighted coronal MRI showed that the lesions were slightly enhanced (Fig. 1C).

Initial investigations revealed that the white blood cell count (normal range: 3,900 11,000) was $21,100 / \mathrm{ml}$, the erythrocyte sedimentation rate (normal range: $0 \sim 10$ ) was 59 $\mathrm{mm} / \mathrm{hr}$, and the C-reactive protein (normal range: 0 0.5) was $20.4 \mathrm{mg} / \mathrm{dl}$. The serum GOT (normal range: 5 40) / GPT (normal range: 5 50) / GGTP (normal range: 12 70) levels were 171/63/106 IU/L, serum total bilirubin (normal range: 0.4 1.4) / direct bilirubin (normal range: 0.1 0.35) were 4.3/1.6 mg/dl, and BUN (normal range: 4.5 23.5) / creatinine (normal range: $0.7 \sim 1.4$ ) were $48 / 1.6 \mathrm{mg} / \mathrm{dl}$.

Enhanced CT scans obtained 7 days after MRI showed an increased amount of pus in bilateral multiple psoas abscesses and multilocular intrapelvic and gluteal abscesses (Fig. 1D-F). Initially, a first-generation cephalosporin was administered intravenously. Due to electrolyte imbalance, hypoalbuminemia with pulmonary edema, and delirium tremens, we chose CT-guided percutaneous catheter drainage instead of open surgical drainage.

On the 4th day after admission, a pig-tail drainage catheter (12 F) was introduced into the left psoas muscle under CT guidance (Fig. 2A). On the 6th day after admission, the flexion contracture of the left hip and knee joint were markedly improved. Initial blood cultures and drained pus cultures demonstrated methicillin-sensitive Staphylococcus aureus. Acid-fast stain and polymerase chain reaction (PCR) for Mycobacterium tuberculosis were negative.

On the 10th day after admission, we introduced an additional CT-guided percutaneous catheter into the right psoas muscle (Fig. 2B). The left and right drains were left in place for 3 weeks and 2 weeks, respectively.

A first-generation cephalosporin was administered intra-
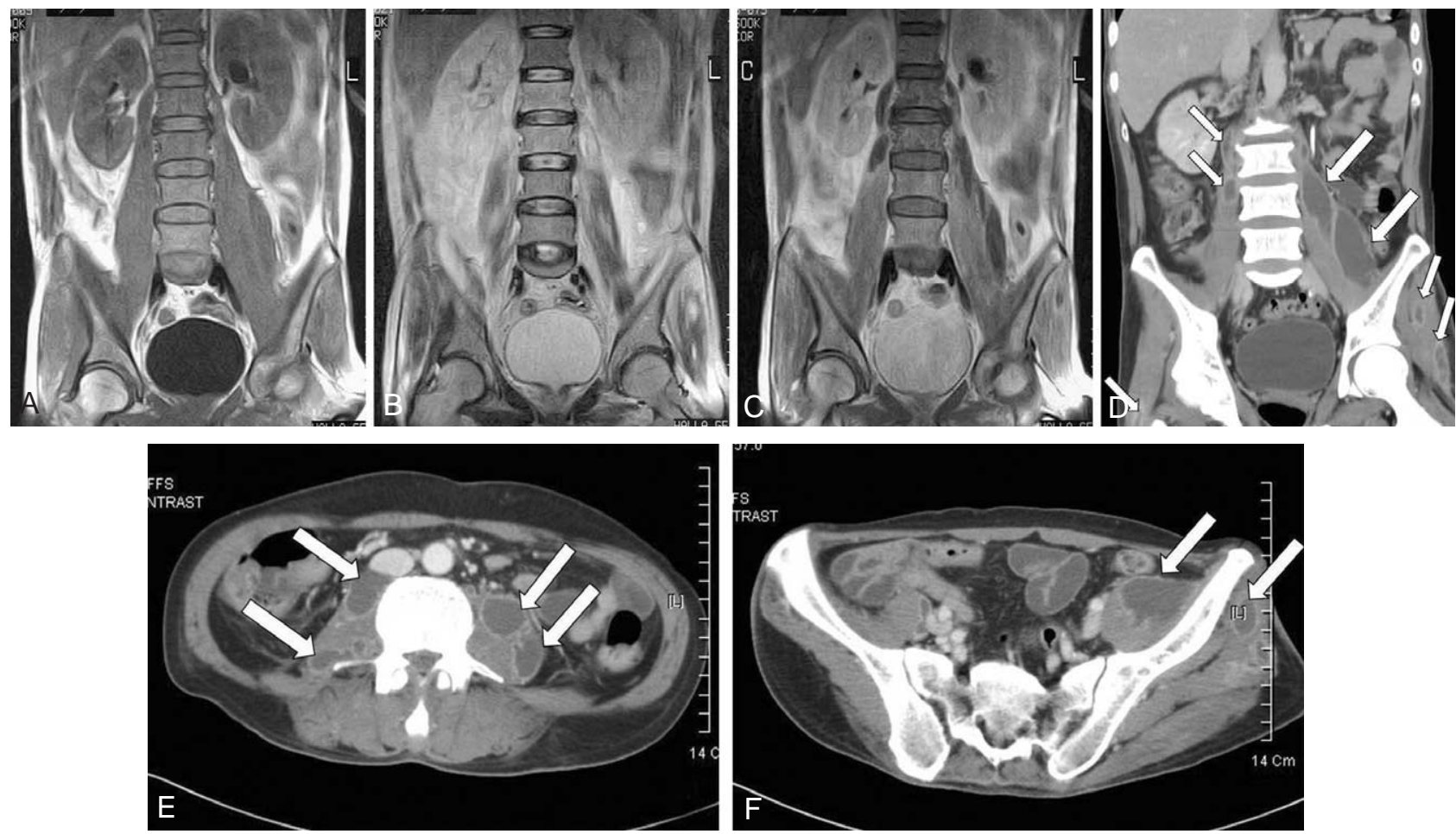

Fig. 1. (A) T1-weighted coronal MRI shows multiple low-signal intensities in both psoas muscles. (B) T2-weighted coronal MRI shows multiple high-signal intensities in both psoas muscles and multiple high-signal bone marrow infiltrations in the L2 and L3 bodies. (C) Enhanced T1-weighted coronal MRI shows slightly enhanced lesions. Enhanced coronal and axial CT scans at 7 days after MRI (D, E, F) show multiple bilateral abscesses in the psoas muscles, multilocular abscesses in the left iliac muscle and gluteal muscle, and an abscess in the right gluteal muscle (white arrows). 

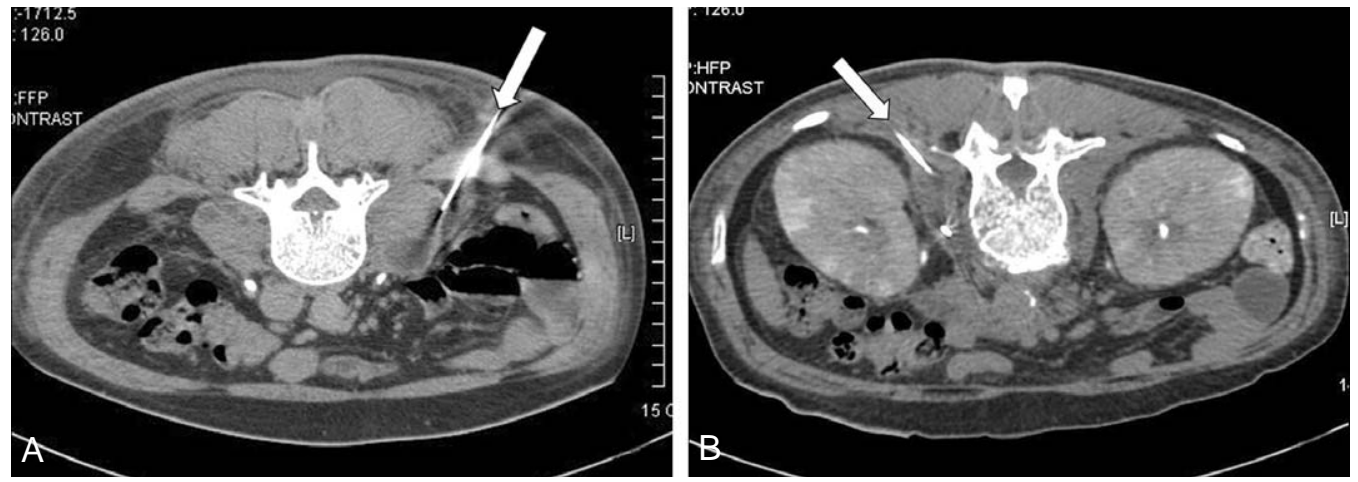

Fig. 2. A percutaneous drainage catheter (white arrow) was introduced into the left psoas muscle under CT guidance (A), and the same procedure (white arrow) was performed for the right psoas muscle (B).

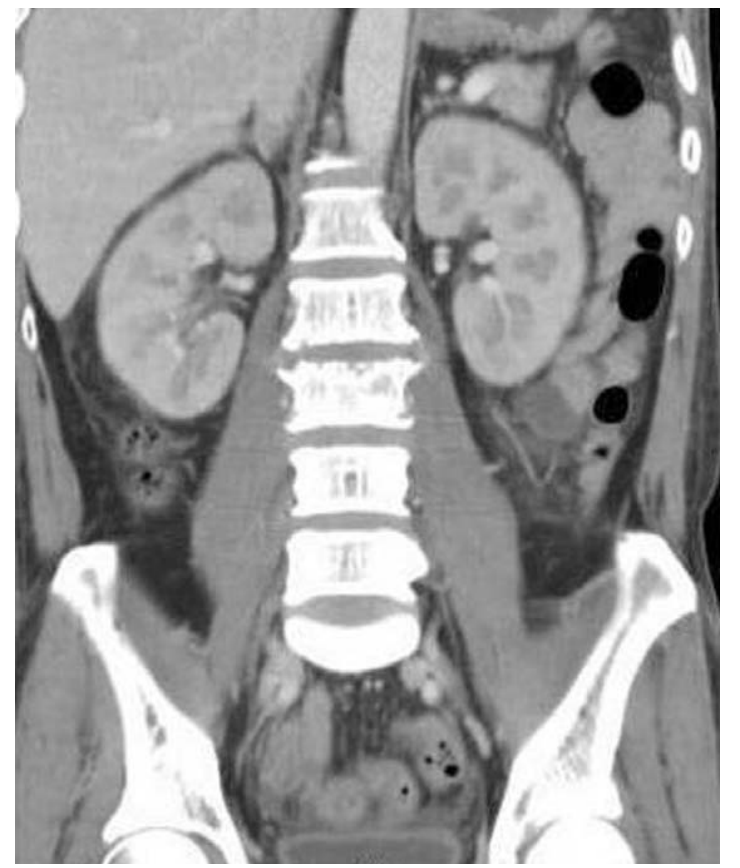

Fig. 3. Three-month follow-up CT scan shows complete resolution of the abscesses. However, irregular disc space narrowing of L2-L3 is noted.

venously for 6 weeks, and an oral cephalosporin was then prescribed for 4 weeks until the CRP and the ESR were normalized. A three-month follow-up CT scan showed complete abscess resolution. However, irregular disc space narrowing of L2-L3 was noted (Fig. 3).

After 2 years of follow-up, the patient returned to normal physical activity without abscess recurrence.

\section{Discussion}

Patients with diabetes mellitus or chronic alcoholism are susceptible to unusual infections. A previous report showed that infection is an important cause of death among patients with diabetes mellitus (about $10 \%)^{3}$. In a diabetic patients with primary psoas abscesses, the major pathogen is Staphylococcus aureus; enteric bacteria are the major cause of secondary abscesses. In our case, the pathogen was methicillin-sensitive Staphylococcus aureus.

A few cases of multiloculated abscesses involving psoas, iliac, pelvic floor, and gluteal regions have been reported, but they have been secondary abscesses from vertebral osteomyelitis or tuberculous arthritis of the sacroiliac joint ${ }^{5}$. Our case was more aggressive than previously reported cases.

Radiologic studies provide a better understanding of the anatomic magnitude of the infection and may suggest an underlying cause. CT has been established as the standard imaging study to assess the characteristics and extent of a given abscess, along with its involvement of adjacent structures ${ }^{6}$.

Psoas abscesses have traditionally been treated with retroperitoneal surgical drainage and appropriate antimicrobial therapy. In recent years, percutaneous drainage under image guidance has become a generally accepted method for treating intraabdominal and retroperitoneal abscesses, including psoas abscesses ${ }^{5-9}$. Low mortality and morbidity are major advantages of CT-guided percutaneous catheter drainage.

It is generally agreed that psoas abscesses with concurrent intraabdominal or retroperitoneal abnormalities and multiloculated abscesses should be treated surgically ${ }^{3}$. However, we chose the CT-guided percutaneous catheter drainage procedure because we suspected multiple organ failure associated with sepsis.

If a psoas abscess is not recognized and treated promptly, the mortality rate is up to $55.6 \%$, with a mortality rate of up 
to $100 \%$ in bilateral psoas abscesses ${ }^{1,3,10}$. We successfully treated the patient using a CT-guided drainage procedure for multiple bilateral psoas abscesses and multilocular intrapelvic and gluteal abscesses. We used only one drainage catheter for each psoas muscle, and the drainage was considered effective. The multiloculated intrapelvic and gluteal abscesses originated from the psoas muscle, and each of the abscesses had an interconnection to the psoas muscle.

A high degree of suspicion is necessary to achieve early diagnosis of psoas abscesses in older diabetic patients with fever, pain in the flank or back, limp, and/or flexion contracture of the hip joint and the knee joint. CT scans are useful in diagnosis, and CT-guided percutaneous catheter drainage with appropriate antibiotic therapy is effective treatment in selected patient. We recommend this procedure in patients with high-risk medical illnesses and multiple abscesses or bilateral abscesses.

\section{REFERENCES}

1. Gruenwald I, Abrahamson J, Cohen O: Psoas abscess: case report and review of the literature. J Urol 1992; 147: 1624-1626.

2. Ricci MA, Rose FB, Meyer KK: Pyogenic psoas abscess: worldwide variations in etiology. World J Surg 1986; 10: 834-843.
3. Kao PF, Tsui KH, Leu HS, Tsai MF, Tzen KY: Diagnosis and treatment of pyogenic psoas abscess in diabetic patient: usefulness of computed tomography and gallium67 scanning. Urology 2001; 57: 246-251.

4. Santaella RO, Fishman EK, Lipsett PA: Primary vs secondary iliopsoas abscess. Presentation, microbiology, and treatment. Arch Surg 1995; 130: 1309-1313.

5. Dinç H, Onder C, Turhan AU, et al: Percutaneous catheter drainage of tuberculous and nontuberculous psoas abscesses. Eur J Radiol 1996; 23: 130-134.

6. Zissin R, Gayer G, Kots E, Werner M, Shapiro-Feinberg M, Hertz M: Iliopsoas abscess: a report of 24 patients diagnosed by CT. Abdom Imaging 2001; 26: 533539.

7. Dinç H, Sari A, Yulug G, Gumele HR: CT-guided drainage of multilocular pelvic and gluteal tuberculous abscesses. AJR Am J Roentgenol 1996; 167: 667-668.

8. Cantasdemir M, Kara B, Cebi D, Selcuk ND, Numan F: Computed tomography-guided percutaneous catheter drainage of primary and secondary iliopsoas abscesses. Clin Radiol 2003; 58: 811-815.

9. Gupta S, Suri S, Gulati M, Singh P: Ilio-psoas abscesses: percutaneous drainage under image guidance. Clin Radiol 1997; 52: 704-707.

10. Huang JJ, Ruaan MK, Lan RR, Wang MC: Acute pyogenic iliopsoas abscess in Taiwan: Clinical features, diagnosis, treatments and outcome. J Infect 2000; 40: 248-255. 